\title{
Preexisting TP53 mutation in therapy-related acute myeloid leukemia
}

\author{
Eduard Schulz • Karl Kashofer • Ellen Heitzer • \\ Ketaki N. Mhatre $\cdot$ Michael R. Speicher • \\ Gerald Hoefler • Heinz Sill
}

Received: 27 July 2014 / Accepted: 9 August 2014 / Published online: 20 August 2014

(C) The Author(s) 2014. This article is published with open access at Springerlink.com

Keywords Therapy-related myeloid neoplasms - TP53 .

Leukemogenesis

\section{Dear Editor,}

Therapy-related myeloid neoplasms (t-MNs) are a unique clinical entity occurring as late complication of chemotherapy and radiotherapy administered for a primary disease [1]. According to the WHO classification, t-MNs are thought to be due to mutational events in hematopoietic stem and precursor cells (HSPCs) induced by these treatments [2]. However, no consistent biomarker has been identified yet that classifies a particular neoplasm as "therapy-related" [3]. This raises the possibility that other mechanisms may also be operational in their pathogenesis. We and others [4], therefore, hypothesized that mutations contributing to leukemic transformation were preexisting in HSPCs of some of these individuals.

In this study, we selected patients with therapy-related AML (t-AML) following cytotoxic treatment of malignant lymphomas as bone marrow (BM) biopsies are routinely

Presented in part as abstract form at the nineteenth annual congress of the European Hematology Association, Milano, Italy, June 12-15, 2014

Electronic supplementary material The online version of this article (doi:10.1007/s00277-014-2191-0) contains supplementary material, which is available to authorized users.

E. Schulz $\cdot$ K. N. Mhatre $\cdot$ H. Sill $(\square)$

Division of Hematology, Department of Internal Medicine, Medical University of Graz, Auenbruggerplatz 38D, A-8036 Graz, Austria

e-mail: heinz.sill@medunigraz.at

K. Kashofer $\cdot$ G. Hoefler

Institute of Pathology, Medical University of Graz,

Auenbruggerplatz 25/1, A-8036 Graz, Austria

E. Heitzer · M. R. Speicher

Institute of Human Genetics, Medical University of Graz,

Harrachgasse 21/VIII, A-8010 Graz, Austria performed during their initial staging procedures. We focused on the TP53 gene which is frequently mutated in t-AMLs exhibiting a potentially important role in leukemogenesis [5-7]. We identified a somatic heterozygous 64-base pair duplication (Fig. 1a) in a 71 year-old male Caucasian patient who suffered from Hodgkin lymphoma 13 years ago treated by chemotherapy and radiotherapy. To search for potential cooperating mutations, we performed Ion Torrent deep sequencing of recurrently mutated genes in AML [8]. However, no further mutations could be identified (see Supplementary Information for list of genes).

We established a highly sensitive PCR assay specific for this rearrangement (Fig. 1b) and could unambiguously demonstrate the presence of the TP53 mutation in the patient's BM obtained at the time of the lymphoma staging (Fig. 1c). Surprisingly, the TP53 duplication was also detected in a reactive lymphadenitis sample obtained 7 years before lymphoma diagnosis (Fig. 1c). To further demonstrate that expansion of the TP53 mutated clone occurred following cytotoxic treatment, we quantified the TP53 duplication by digital PCR (dPCR) which showed that the relative proportion of mutated cells increased substantially in the t-AML specimen (Fig. 1d). It, furthermore, confirmed the TP53 duplication being a somatically acquired event as it was absent from a skin biopsy obtained at the time of the leukemia diagnosis.

In the case report presented here, we were able to demonstrate that cytotoxic treatment did not induce a leukemiaspecific mutation but rather may have facilitated the expansion of a pre-leukemic clone harboring a somatic TP53 mutation. Since dPCR data quantifying the TP53 duplication were comparable in lymph node and pretreatment BM, the mutation might have occurred in HSPCs that retained their lymphoid as well as myeloid differentiation potential and remained dormant for many years. This finding challenges current concepts of therapy-related leukemogenesis and is in line with data presented at the 2013 annual meeting of the American Society 
A

TP53 c.276_339dup (p.Leu114Profs³1)

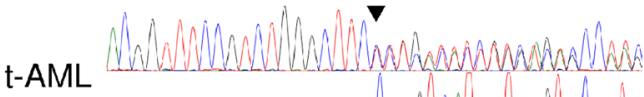

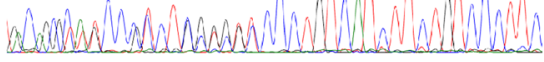

Skin

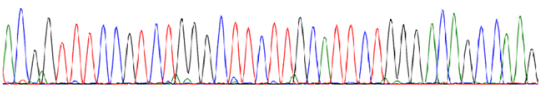

B

62 bp PCR product

$$
\langle\sqrt{n \backslash t w w}\rangle
$$

$5^{\prime}$
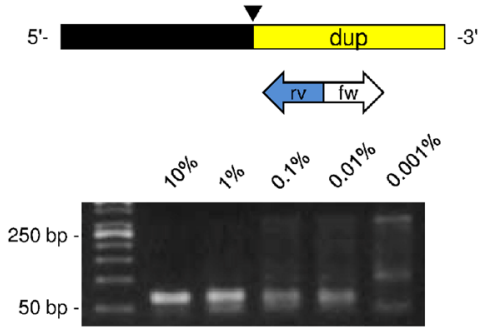

C

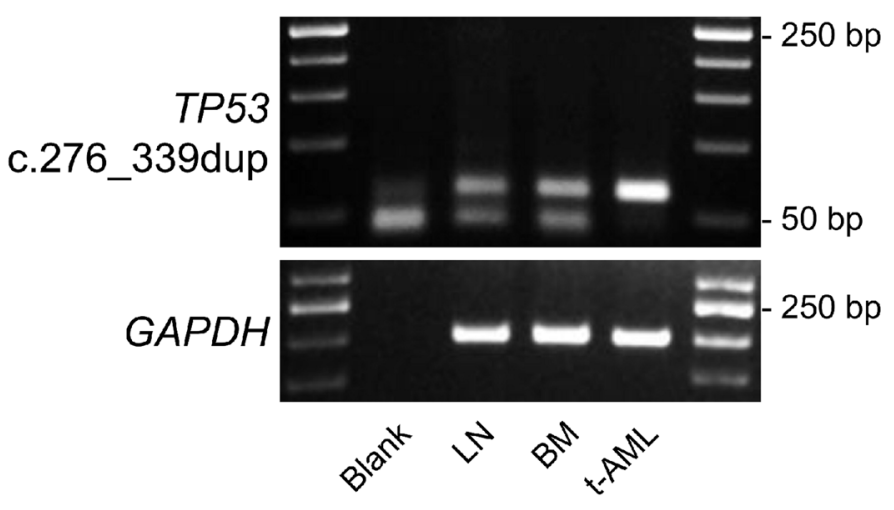

D

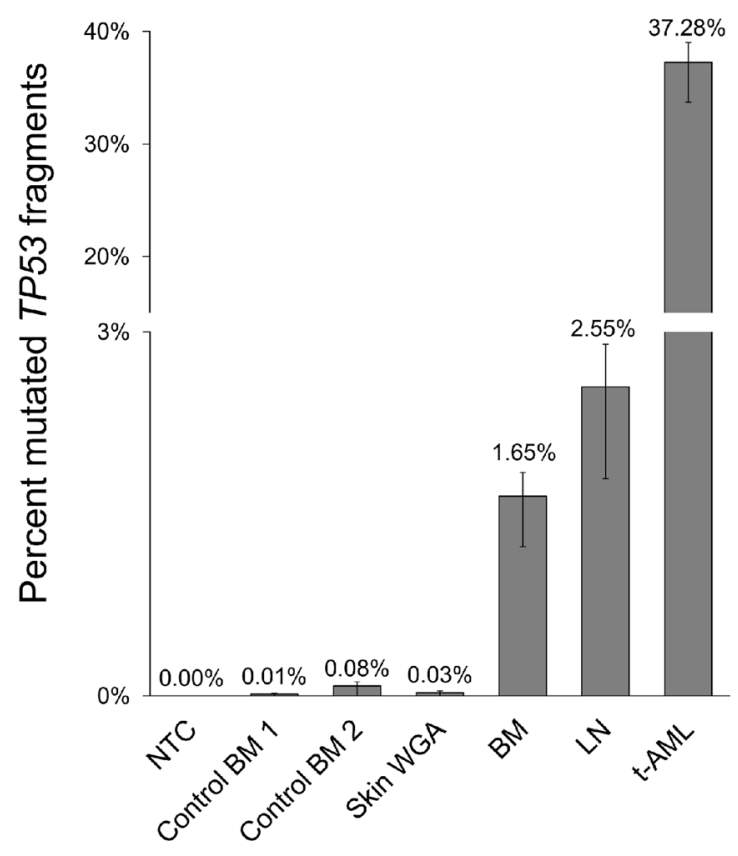


4 Fig. 1 Detection of a preexisting TP53 mutation in a patient with therapy-related acute myeloid leukemia (t-AML). a Bi-directional Sanger sequencing demonstrates the leukemia-specific $64 \mathrm{bp}$ duplication in exon 4 of the TP53 gene. b The primer pair enables the detection of the duplication to a dilution of $0.01 \%$ t-AML DNA in normal control DNA. Analysis of four AML control samples revealed the absence of this duplication in all of them (data not shown). $\mathbf{c}$ The 62base pair product (upper band) specific for the TP53 duplication could be detected in the bone marrow (BM) obtained at diagnosis of Hodgkin lymphoma as well as in a lymphadenitis specimen (LN) the latter obtained 20 years before diagnosis of t-AML. The lower band refers to primer dimers, GAPDH (lower gel) served as control gene for DNA integrity. d Expansion of cells with the TP53 duplication determined by digital PCR. Relative ratios between mutant TP53 fragments and BRAF wild-type fragments were assessed using the QuantStudio 3D Digital PCR System (Life Technologies, Carlsbad, CA). Copy number changes at the $B R A F$ locus of the t-AML were excluded using low coverage whole genome sequencing to ensure that $B R A F$ is suitable as a reference and not subjected to copy number changes. Data shown are means of assays performed in duplicates and error bars indicate $95 \%$ confidence intervals. $B M$ bone marrow; $L N$ lymphadenitis sample; $N T C$ no template control; $W G A$ whole genome amplified DNA

of Hematology [4]. There, somatic TP53 variants could be identified at low frequencies in mobilized peripheral blood leukocytes of two t-MDS/t-AML cases years before diagnosis. However, in clinical practice, HSPC harvests from peripheral blood are performed following intense chemotherapy including application of recombinant granulocyte-colony factor. Here, we provided definitive evidence that a leukemiaspecific mutation could be found in HSPCs before any cytotoxic treatment was administered.

\begin{abstract}
Acknowledgment The work was funded in part by the Austrian National Bank, Anniversary Fund (grant no. 13918), Land Steiermark, Leukämiehilfe Steiermark and "Vereinigung Forschungsförderung" at Medical University of Graz, Austria. E.S. is supported by a dissertational grant from the Austrian Society of Hematology and Oncology.
\end{abstract}

Ethical standards statement The study was approved by the ethics committee of the Medical University of Graz, Austria, and written informed consent was obtained from all patients.

\footnotetext{
Authorship E.S., K.K., E.H., M.R.S., G.H. and H.S. conceived experiments. K.M. and E.S. performed Sanger sequencing. K.K. performed and analyzed targeted deep sequencing. E.H. performed and analyzed dPCR. G.H. and H.S. provided patient samples. E.S. and H.S. had full access to all of the data in the study and take responsibility for their integrity and the accuracy of the data analysis. E.S. and H.S. wrote the manuscript which was approved by all authors.
}

Conflict of interest The authors declare no competing financial interests.

Open Access This article is distributed under the terms of the Creative Commons Attribution License which permits any use, distribution, and reproduction in any medium, provided the original author(s) and the source are credited.

\section{References}

1. Churpek JE, Larson RA (2013) The evolving challenge of therapyrelated myeloid neoplasms. Best Pract Res Clin Haematol 26(4):309317. doi:10.1016/j.beha.2013.09.001

2. Vardiman JW, Arber DA, Brunning RD, Larson RA, Matutes E, Baumann I et al (2008) Therapy-related myeloid neoplasms. In: Swerdlow SH, Campo E, Harris NL, Jaffe ES, Pileri SA, Stein H (eds) WHO classification of tumours of haematopoietic and lymphoid tissues, 4th edn. IARC, Lyon, pp 127-129

3. Mauritzson N, Albin M, Rylander L, Billstrom R, Ahlgren T, Mikoczy Z, Bjork J, Stromberg U, Nilsson PG, Mitelman F, Hagmar L, Johansson B (2002) Pooled analysis of clinical and cytogenetic features in treatment-related and de novo adult acute myeloid leukemia and myelodysplastic syndromes based on a consecutive series of 761 patients analyzed 1976-1993 and on 5098 unselected cases reported in the literature 1974-2001. Leukemia 16(12):2366-2378. doi:10.1038/ sj.leu. 2402713

4. Wong TN, Ramsingh G, Young A, Shen D, Miller C, Lamprecht T, Heath S, Fulton RS, Mardis ER, Ding L, Westervelt P, Welch J, Walter MJ, Graubert T, DiPersio JF, Ley TJ, Druley TE, Wilson RK, Link DC (2013) The role of early TP53 mutations on the evolution of therapyrelated AML. Blood 122(21):5

5. Shih AH, Chung SS, Dolezal EK, Zhang SJ, Abdel-Wahab OI, Park CY, Nimer SD, Levine RL, Klimek VM (2013) Mutational analysis of therapy-related myelodysplastic syndromes and acute myelogenous leukemia. Haematologica 98(6):908-912. doi:10.3324/haematol. 2012.076729

6. Schulz E, Valentin A, Ulz P, Beham-Schmid C, Lind K, Rupp V, Lackner H, Wolfler A, Zebisch A, Olipitz W, Geigl J, Berghold A, Speicher MR, Sill H (2012) Germline mutations in the DNA damage response genes BRCA1, BRCA2, BARD1 and TP53 in patients with therapy related myeloid neoplasms. J Med Genet 49(7):422-428. doi: 10.1136/jmedgenet-2011-100674

7. Zhao Z, Zuber J, Diaz-Flores E, Lintault L, Kogan SC, Shannon K, Lowe SW (2010) P53 loss promotes acute myeloid leukemia by enabling aberrant self-renewal. Genes Dev 24(13):1389-1402. doi: $10.1101 / \mathrm{gad} .1940710$

8. Cancer Genome Atlas Research Network (2013) Genomic and epigenomic landscapes of adult de novo acute myeloid leukemia. $\mathrm{N}$ Engl J Med 368(22):2059-2074. doi:10.1056/NEJMoa1301689 